\title{
Structural Determinants of Specific Lipid Binding to Potassium Channels
}

\author{
Markus Weingarth, ${ }^{\dagger}$ Alexander Prokofyev, ${ }^{\dagger,}$ Elwin A. W. van der Cruijsen, ${ }^{\dagger}$ Deepak Nand, ${ }^{\dagger}$
} Alexandre M. J. J. Bonvin, ${ }^{\dagger}$ Olaf Pongs, ${ }^{\dagger}$ and Marc Baldus ${ }^{*}{ }^{\dagger}$

${ }^{\dagger}$ Bijvoet Center for Biomolecular Research, Faculty of Science, Utrecht University, Padualaan 8, $3584 \mathrm{CH}$ Utrecht, The Netherlands

${ }^{\ddagger}$ Saarland University, Faculty of Medicine, Department of Physiology, Building 59, 66421 Homburg, Germany

\section{Supporting Information}

ABSTRACT: We have investigated specific lipid binding to the pore domain of potassium channels KcsA and chimeric KcsAKv1.3 on the structural and functional level using extensive coarse-grained and atomistic molecular dynamics simulations, solid-state NMR, and single channel measurements. We show that, while KcsA activity is critically modulated by the specific and cooperative binding of anionic nonannular lipids close to the channel's selectivity filter, the influence of nonannular lipid binding on KcsA-Kv1.3 is much reduced. The diminished impact of specific lipid binding on KcsA-Kv1.3 results from a
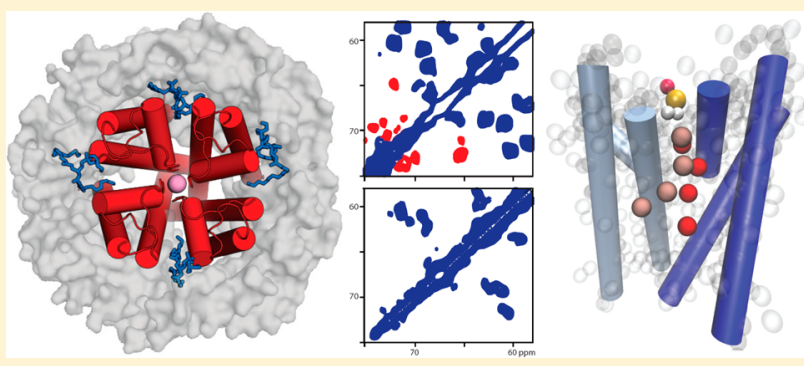
point-mutation at the corresponding nonannular lipid binding site leading to a salt-bridge between adjacent KcsA-Kv1.3 subunits, which is conserved in many voltage-gated potassium channels and prevents strong nonannular lipid binding to the pore domain. Our findings elucidate how protein-lipid and protein-protein interactions modulate $\mathrm{K}^{+}$channel activity. The combination of $\mathrm{MD}, \mathrm{NMR}$, and functional studies as shown here may help to dissect the structural and dynamical processes that are critical for the functioning of larger membrane proteins, including $\mathrm{Kv}$ channels in a membrane setting.

\section{INTRODUCTION}

Structure and function of many membrane proteins are crucially modulated by direct interaction with lipid residues. $^{1-9}$ These lipids are typically categorized as annular, constituting a shell around the protein and showing relatively weak binding affinity, or as nonannular. The latter are specific lipid residues that bind with high affinity to hydrophobic clefts of membrane proteins, often copurify alongside the protein, and are resolved in crystal structures. For example, MacKinnon et al. revealed the presence of a diacylglycerol fragment in the crystal structure of the homotetrameric potassium channel KcsA from Streptomyces lividans that requires negatively charged lipids for activity. ${ }^{10}$ The location of the lipid fragment in a shallow groove between adjacent channel subunits in proximity to the two positively charged arginine residues R64 and R89 (Figure S1) would thus be compatible with a prominent influence of nonannular anionic lipid binding on channel activity. ${ }^{10}$ Much research has since been invested to elucidate the nature and affinity of the lipid residues involved in nonannular lipid binding, ${ }^{11-13}$ although how and if specific lipid residues modulate the activity of the KcsA channel remain elusive.

KcsA with its inner helix bundle serves as an archetypical pore domain of voltage-gated $(\mathrm{Kv})$ potassium channels, which thus potentially share KcsA's extracellular nonannular site. ${ }^{14}$ Interestingly, the positively charged residue R64 in KcsA is replaced by a negatively charged residue at the equivalent position in many $\mathrm{Kv}$ channels, particularly in human Kv1.x
(KNCA) and Kv7.x (KCNQ) (Figure 1), although this charge inversion is not conserved throughout the whole $\mathrm{KCNx}$ family.

A better understanding of nonannular lipid binding to the pore domain is of general importance to $\mathrm{Kv}$ channels. Indeed, specific binding of spingomyelin involving the pore domain was

\begin{tabular}{|c|c|c|c|c|c|c|c|c|c|c|c|c|}
\hline \multirow[b]{2}{*}{$K \operatorname{cs} A$} & \multicolumn{7}{|c|}{64} & \multicolumn{5}{|c|}{89} \\
\hline & $\mathrm{T}$ & $Y$ & $P$ & $\mathrm{R}$ & A & L & W & L W & G & $\mathrm{R}$ & V & A \\
\hline$K v A P$ & $S$ & V & $F$ & $D$ & A & L & W & P I & G & K & V & $\mathrm{G}$ \\
\hline$h K v 1.1$ & S & I & P & $D$ & A & $F$ & W & G & G & K & 1 & G \\
\hline$h K v 1.2$ & S & I & $P$ & D & A & $F$ & W & V W & G & K & 1 & G \\
\hline$h K v 1.3$ & $S$ & I & $P$ & $D$ & A & $F$ & W & I G & G & K & 1 & G \\
\hline$h K v 1.4$ & $S$ & I & P & $D$ & A & $F$ & W & $V G$ & G & K & 1 & A \\
\hline$h K v 1.5$ & $S$ & I & P & D & A & $F$ & W & L W & $G$ & K & L V & A \\
\hline$h K v 1.6$ & S & I & $P$ & D & A & $F$ & W & $V G$ & $G$ & $\mathrm{~K}$ & I & $\mathrm{G}$ \\
\hline$h K v 1.7$ & $S$ & I & $P$ & $E$ & $S$ & $F$ & W & $V G$ & $G$ & $\mathrm{~K}$ & I & G \\
\hline$h K v 7.1$ & $S$ & I & P & D & A & L & W & $W W$ & G & K & $\mathrm{T}$ & A \\
\hline$h K v 7.2$ & $\mathrm{~T}$ & $Y$ & A & $D$ & A & $\mathrm{L}$ & W & $\mathrm{W} N$ & G & $R$ & L & A \\
\hline$h K v 7.3$ & $\mathrm{~T}$ & $\mathrm{Y}$ & A & D & A & L & W & $W E$ & G & $R$ & L & A \\
\hline$h K v 7.4$ & $S$ & $Y$ & A & D & $S$ & $\bar{L}$ & W & $W L$ & $G$ & $\mathrm{R}$ & V & A \\
\hline$h K v 7.5$ & $\mathrm{~T}$ & $Y$ & A & D & A & & W & W L & G & $\mathrm{R}$ & L & A \\
\hline
\end{tabular}

Figure 1. Sequence alignment of KcsA and a series of $\mathrm{Kv}$ channels. The positively charged residue pair R64 and R89 of KcsA's extracellular nonannular site is highlighted. It is shown that R64 is replaced by a negatively charged residue in many $\mathrm{Kv}$ channels.

Received: December 6, 2012

Published: February 20, 2013 
a)

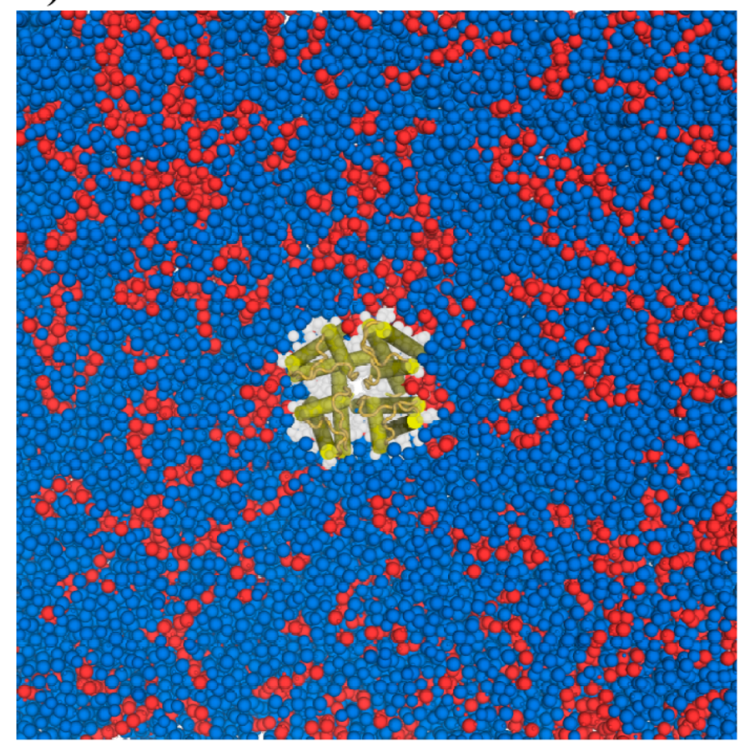

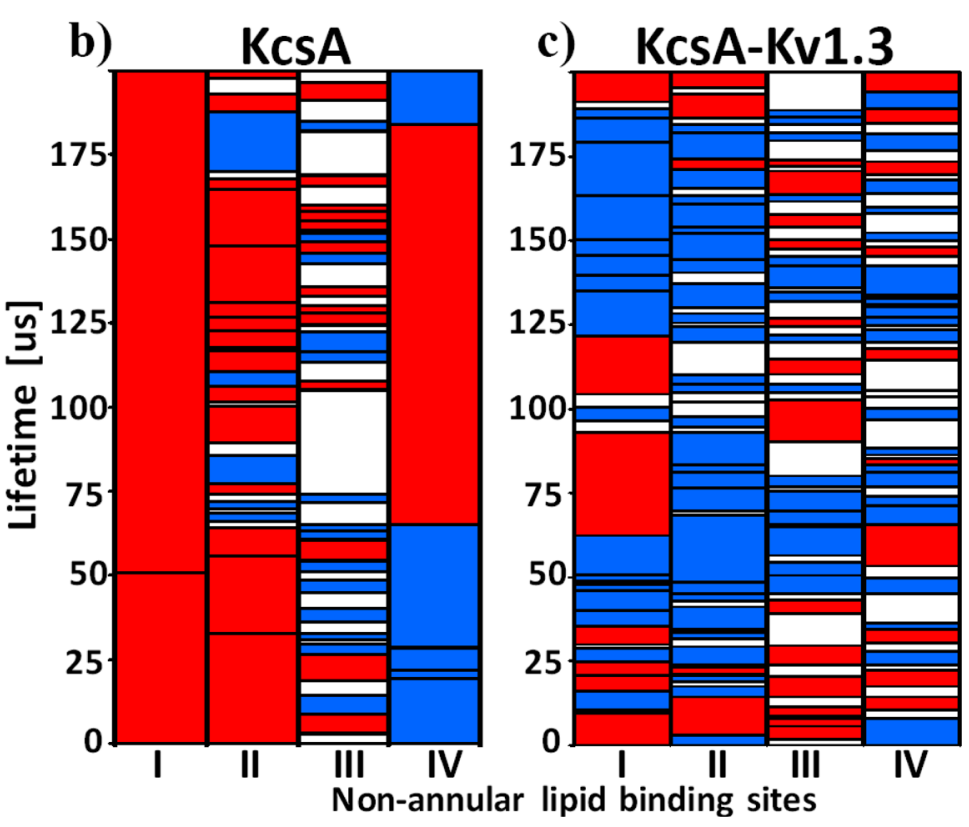

Figure 2. (a) The system used for the CGMD simulations: KcsA/KcsA-Kv1.3 channels were inserted in a large $\left(\sim 25 \times 25\right.$ nm $\left.{ }^{2}\right)$ mixed DPPC:DPPG bilayer (3:1 molar ratio). DPPC lipids are highlighted in blue and DPPG lipids in red. The atomic-resolution channel structure is superimposed on its CG representation. (b,c) Evaluation of the lifetimes of bound DPPC (blue) and DPPG (red) for each nonannular site of (b) KcsA and (c) KcsA-Kv1.3 over trajectories of $210 \mu$ s. Each blue or red box in the four columns represents a single binding event. The first $10 \mu$ s were considered as equilibration period. Periods in which the lifetimes did not exceed $2 \mu$ s are indicated in white. During these white periods, the binding sites were usually occupied by DPPC or DPPG in fast exchange and rarely empty. The nonannular sites are plotted in a clockwise order, i.e., grooves I and II are opposite to grooves III and IV, respectively. The numbering of the binding sites is arbitrary.

shown to have a prominent influence on $\mathrm{Kv}$ channel voltage dependence and gating. ${ }^{15}$ Here we have compared the influence of specific lipid binding in KcsA to the chimeric KcsA-Kv1.3, which contains 11 residues of the human Kv1.3 in the turret region, ${ }^{16,17}$ including mutation R64D (Figures 1 and S2). In a combined approach of extensive coarse-grained molecular dynamics (CGMD) $)^{18,19}$ simulations on the submillisecond time scale, solid-state NMR (ssNMR), and electrophysiological measurements, we show that nonannular anionic lipid binding in proximity of the selectivity filter correlates with the lipid sensitivity of KcsA activity. In KcsA-Kv1.3, the presence of a salt-bridge at the corresponding nonannular lipid binding site of $\mathrm{Kv}$ channels decreases residence times of bound lipids by about 1 order of magnitude. Based on these insights and supported by atomistic MD simulations, we formulate a molecular mechanism how negatively charged lipids could modulate KcsA's activity and discuss the role of a salt-bridge between S5 and S6 helices on $\mathrm{Kv}$ channel activity in general.

\section{RESULTS AND DISCUSSION}

Structural Studies: MD. Several studies, especially by fluorescence spectroscopy ${ }^{11}$ and electrophysiological measurements, ${ }^{13}$ have confirmed a general preference of anionic over zwitterionic lipids for the extracellular nonannular site of KcsA. Yet, so far, atomic insights have been exclusively brought about by atomistic MD simulations over a length of $20-30 \mathrm{~ns},{ }^{20}$ excluding the possibility to monitor lipid binding on the microto millisecond time scale. We sought to bridge the time scale gap between experimental and in silico studies by resorting to large-scale CGMD simulations over a total effective simulation time of $0.63 \mathrm{~ms}(2 \times 0.21 \mathrm{~ms}$ for KcsA, $0.21 \mathrm{~ms}$ for KcsA-

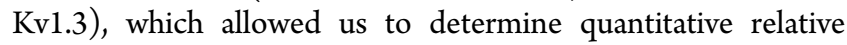
affinities for zwitterionic and anionic lipids. Simulations were carried out in mixed DPPC:DPPG bilayers (3:1 molar ratio)
(Figure 2a), which roughly correspond to the molar ratio of zwitterionic to anionic lipids in the inner membrane of E. coli, ${ }^{21}$ in which both channels were expressed for the experimental part of this study. The CGMD simulations revealed considerable differences in nonannular lipid binding between KcsA and KcsA-Kv1.3. We observed for both channels nonannular lipid binding to the intersubunit binding site on the microsecond time scale, however, the lifetime of nonannular DPPG was about 1 order of magnitude longer for KcsA (Figure 2b,c). For KcsA, averaged over a total simulation time of $420 \mu \mathrm{s}$ (Figure S3), we found lifetimes (without counting for site III) for DPPG and DPPC of 31.8 and $11.6 \mu$ s, respectively, while we observed lifetimes for DPPG and DPPC of 5.9 and 4.9 $\mu \mathrm{s}$, respectively, in KcsA-Kv1.3. A summation over all lifetimes gave rise to an absolute and relative (relative means assuming a 1:1 DPPC:DPPG molar ratio) DPPG occupancy of the nonannular sites of 64 and $84 \%$ in KcsA, respectively, which agrees well with fluorescence measurements. ${ }^{1,22}$ In KcsAKv1.3, however, the preference for anionic lipids is much lower with 37 and 64\% absolute and relative DPPG occupancy, respectively. Preliminary GCMD simulations with the KcsA channel embedded in a 3:1 DOPC:DOPG mixture point to an analogous strong preference of anionic over zwitterionic nonannular lipid binding.

Interestingly, the CGMD simulations point to cooperativity in the nonannular lipid binding to KcsA. Lipids bound strongly to two sites (sites I and IV in Figure 2b) with lifetimes of more than $100 \mu \mathrm{s}$, with moderate affinity to one site (site II) and with little affinity to another binding site (site III). This behavior could be reproduced in a second KcsA simulation (Figure S3) and matches well with fluorescence and single channel conductance measurements, ${ }^{12}$ which found cooperative behavior in the effect of anionic lipids on channel open probability. This led to a model in which the occupation of three of the four 
nonannular sites by anionic lipids sufficed for channel activity. ${ }^{12}$ Remarkably, MacKinnon and coworkers measured a ratio of 0.7 molecules of copurified lipid per KcsA monomer, ${ }^{10}$ which would also be consistent with strong to moderate binding to only three sites, ${ }^{10}$ as the simulations suggest. The cooperative lipid binding, which we observe in the CG simulations seems to be modulated by the bulky side chains of P63 and L86 of adjacent subunits, which may prevent lipid headgroups to approach R64 and R89. Remarkably, the onset of this scenario is also visible in atomistic simulations (Figure S4), which show the narrowing of one nonannular binding pocket by the "lock" P63-L86, which impedes headgroup anchoring and binding of the $s n 1$ lipid chain to the groove between the subunits, while the other three nonannular sites remain accessible.

An analysis of the trajectories revealed the formation of a tight salt-bridge D64-R89 between all adjacent subunits of KcsA-Kv1.3 (Figure 3a), which has a major influence on

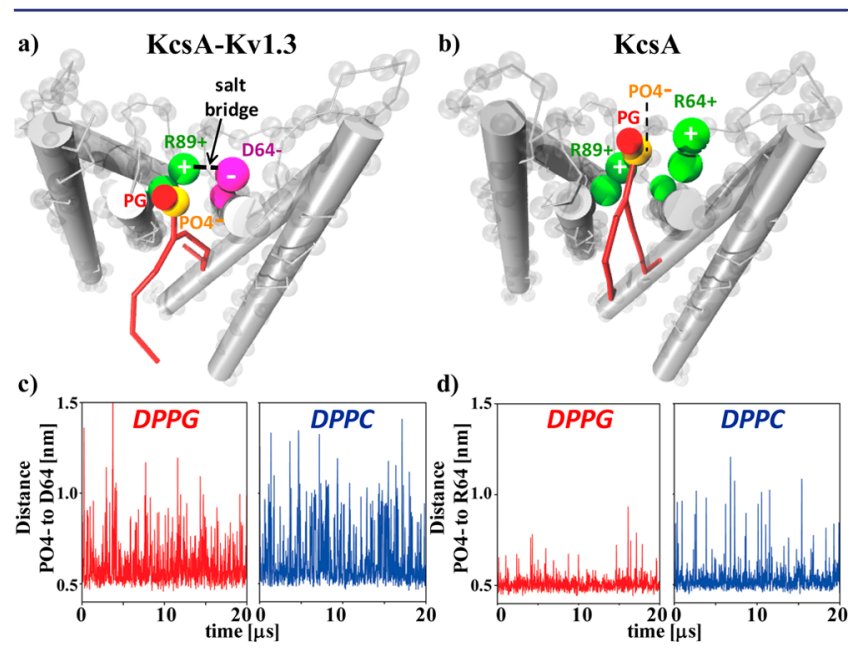

Figure 3. Schematic representation of nonannular lipid binding to (a) KcsA-Kv1.3 and (b) KcsA. Backbone beads are highlighted as gray spheres. $(c, d)$ Trajectories of the distance of the phosphate-groups of bound DPPG (red) and DPPC (blue) to (c) D64 of KcsA-Kv1.3 and (d) R64 of KcsA. The minimum distance of two CG beads is $0.5 \mathrm{~nm}$. In KcsA-Kv1.3, narrowing of the nonannular site by salt-bridge D64R89 and electrostatic repulsion between D64 and lipid-phosphate groups prevents headgroup anchoring, whereas phosphate groups are ideally sandwiched by R64 and R89 in KcsA.

specific lipid binding. On the one hand, the salt-bridge narrows the nonannular site, and on the other hand, D64 repulses phosphate groups of any bound lipid (Figure 3a,c). This scenario impedes lipid headgroup anchoring on top of KcsA-

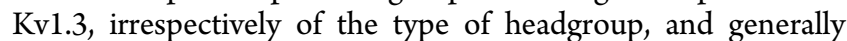
leads to reduced nonannular lipid lifetimes in comparison to KcsA (Figure 3b). In KcsA the positively charged R64 and R89 can ideally sandwich negatively charged lipid-phosphate groups (Figure $3 \mathrm{~b}, \mathrm{~d}$ ). This shielding of the two arginine residues by the presence of lipid-phosphate groups in fact stabilizes the KcsA structure. The predominant influence of the charge of residue 64 on nonannular lipid residence times was verified by a CGMD simulation of a R64D-KcsA mutant $>50 \mu \mathrm{s}$. The results of this simulation showed lipid residence times similar to KcsAKv1.3. Moreover, in KcsA-Kv1.3, lipid headgroups do only moderately modulate specific lipid binding (Figure 3c), since both positively charged PC and negatively polarized PG headgroups encounter an attractive and a repulsive partner in the D64-R89 pair. In KcsA, however, the headgroup has a major influence on specific lipid binding. While PC headgroups are repulsed by residues R64 and R89, PG headgroups are attracted and remain tightly bound, which explains the much longer lifetimes for DPPG (Figure 3d). Note that the description of the headgroups as charged beads is presumably a good characterization of nonannular lipid binding to KcsA, which seems to be sensitive to the headgroup's charge, however not to its exact structure. ${ }^{11,22}$

Structural Studies: Solid-State NMR. The CGMD simulations indicate weaker nonannular lipid binding to KcsA-Kv1.3 as a direct consequence of salt-bridge D64-R89. Notably, also the crystal structure of bacterial channel KvAP, ${ }^{26}$ solved in detergent micelles, suggests a salt-bridge between residues D185 and $\mathrm{K} 210$, which would be the equivalent to interaction D64-R89. We used our recently suggested strategy $^{27}$ to validate MD simulations by tailored ssNMR experiments to probe whether this salt-bridge is conserved in KcsA-Kv1.3 in the presence of lipid bilayers. We performed 2D ${ }^{13} \mathrm{C}-{ }^{13} \mathrm{C}$ rotational resonance ${ }^{23}$ experiments on KcsA-Kv1.3 reconstituted in asolectin liposomes, for which ssNMR assignments of the residues D64 and R89 have been reported previously. ${ }^{17}$ As shown in Figure 4a, these experiments unequivocally confirmed the presence of key interaction D64-R89 in line with our computational findings.

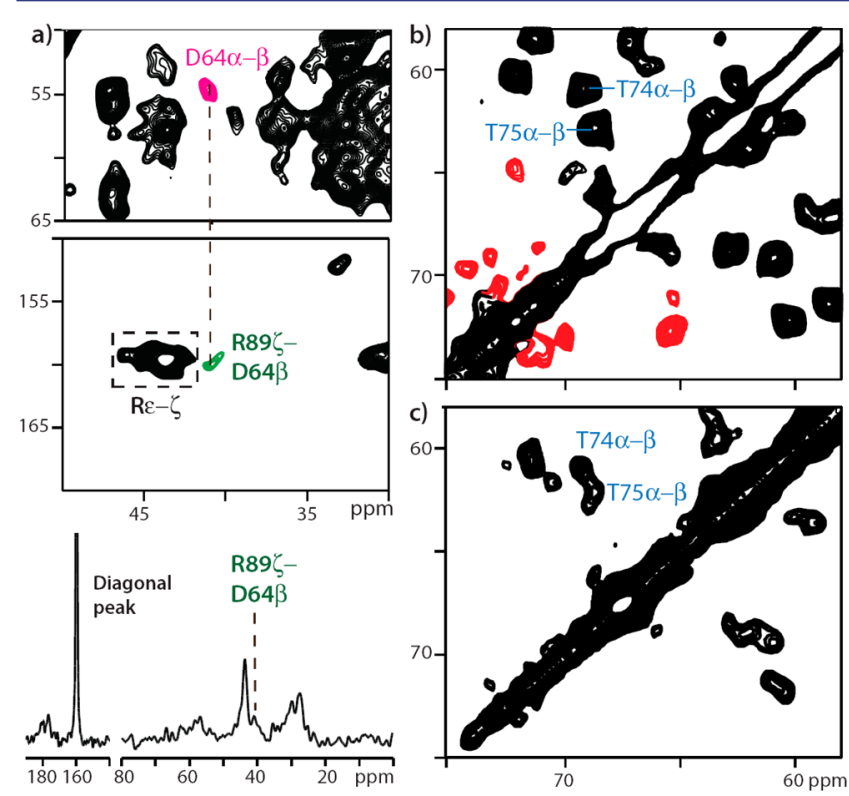

Figure 4. (a) (top) A $2 \mathrm{D}^{13} \mathrm{C}-{ }^{13} \mathrm{C}$ ssNMR rotational resonance ${ }^{23}\left(R^{2}\right)$ experiment confirmed the presence of salt-bridge D64-R89 in KcsAKv1.3. (bottom) Cross-section along $\omega_{2}$ at $\omega_{1}=159.9 \mathrm{ppm}$. (b,c) $2 \mathrm{D}$ ssNMR PARIS ${ }^{24,25}{ }^{13} \mathrm{C}-{ }^{13} \mathrm{C}$ spin diffusion measurements were performed on (b) KcsA and (c) LVWY reverse-labeled KcsAKv1.3 ${ }^{17}$ to probe for tightly bound lipids, copurified from E. coli. The mixing time was $30 \mathrm{~ms}$. Lipid headgroup cross-peaks are shown in red. Protein cross-peaks characteristic for the channels' conductive state are assigned. ${ }^{17}$

Furthermore, we used ssNMR 2D PARIS ${ }^{24,25}{ }^{13} \mathrm{C}-{ }^{13} \mathrm{C}$ experiments to better characterize the properties of nonannular lipid binding to KcsA and KcsA-Kv1.3, which were both reconstituted in asolectin liposomes. For KcsA, we observed cross-peaks between 60 and $80 \mathrm{ppm}$ (Figure $4 \mathrm{~b}$ ), which is the fingerprint region of lipid headgroups, consistent with specifically bound POPG (Figure $4 \mathrm{~b}$ ). ${ }^{28}$ Note that all serine 
and threonine residues, which are the only amino acids that show intraresidual correlations in this region, have been previously assigned. ${ }^{17,29}$ Since the asolectin lipids were not ${ }^{13} \mathrm{C}$-labeled, the lipid cross-peaks are indicative for the presence of tightly bound lipids, copurified from the E. coli inner membrane.

Furthermore, these ${ }^{13} \mathrm{C}-{ }^{13} \mathrm{C}$ lipid cross-peaks imply lipid binding times in the microsecond range or longer but exclude shorter lifetimes and are thus in agreement with the CGMD simulations. Remarkably, while we could detect correlations of copurified lipids in the KcsA spectrum, these correlations were either entirely missing or much weaker for KcsA-Kv1.3 (Figure $4 c)$. The results are consistent with the CGMD simulation data showing decreased nonannular lipid residence times for KcsAKv1.3 (Figure 3c,d). Note that even if copurified ${ }^{13} \mathrm{C}$-labeled lipids would dissociate from the channel after the sample purification process, their presence in the KcsA spectrum is still indicative for stronger nonannular lipid binding. It is important to point out that the differences in binding of copurified lipids between the channels in the ssNMR spectra very likely directly relate to the extracellular nonannular site because KcsA and KcsA-Kv1.3 share the same residues on the cytoplasmic side and differ only in a small number of residues on the extracellular side (Figure S2). Note that the detection of lipid headgroups by ssNMR is not in contradiction to crystallographic data, which could not resolve the headgroup of nonannular lipids, ${ }^{10}$ because the NMR time scale allows observation of more mobile fragments. Partial disorder of the bound lipid headgroups may also impede their resolution in KcsA crystal structures. ${ }^{10}$ In line with such a notion, ssNMR spectra on KcsA are consistent with at least two lipid-spin systems (Figure 4b).

Possible Implications for KcsA Activity. It has been reported in several studies that anionic lipids modulate KcsA's open probability. ${ }^{10,12}$ However, the question of how nonannular anionic lipids modulate KcsA remains unanswered. Here we propose a model which is further supported by atomistic simulations, which would reflect how anionic nonannular lipid binding influences KcsA activity. In addition to the lipid effect on the channel open probability, ${ }^{10,12}$ the low open probability of KcsA under steady-state conditions has been attributed to a relatively swift transition from the opened to the inactivated state. ${ }^{12,30}$ The transition to the inactivated state in KcsA, which shares analogy with C-type inactivation in $\mathrm{Kv}$ channels, ${ }^{30,31}$ comes along with molecular rearrangements within the selectivity filter. ${ }^{30-33}$ Both the nonannular binding site discussed here and the selectivity filter are located on the extracellular channel side. It has been demonstrated that the selectivity filter in KcsA is regulated by a hydrogen-bonding network, especially involving a triad formed by residues E71D80-W67..$^{30,31,34,35}$ Remarkably, mutation E71A in KcsA, which disrupts interaction E71A-D80, also changes the B-factors of R64 in comparison to the wild-type channel, which could imply that the flipped D80 in the E71A mutant directly or indirectly interacts with R64. ${ }^{30}$ Consistent with this idea is the observation that C-type inactivation is significantly slowed down in the KcsA mutant R64A. ${ }^{30}$

We used atomistic $\mathrm{MD}$ simulations to examine potential effects of R64 and R89 on the triad E71-D80-W67. Indeed, in zwitterionic lipids, neither R64 nor R89 markedly interacted with lipids headgroups but turned toward the protein interior, interacting with the carboxyl group of D80, weakening or even disrupting the triad E71-D80-W67 (Figures 5a,b and S5). In the

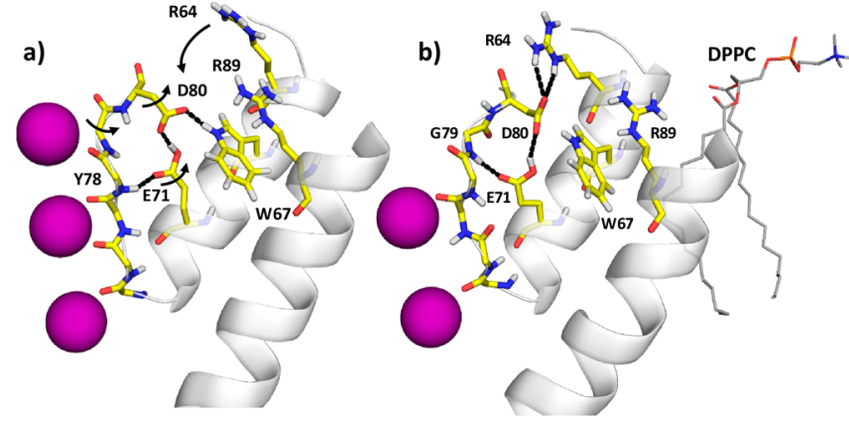

Figure 5. (a) Snapshot of an atomistic MD simulation of KcsA in zwitterionic lipids (DPPC) showing triad E71-D80-W67, which is pivotal to the inactivation process in KcsA. (b) Since DPPC headgroups do not tightly interact with R64 or R89 side chains (see Figure S5 for R89), both side chains turn toward the protein interior, disturbing the triad, which is an important component of KcsA gating.

simulations, the reorientation of the carboxyl group of D80 furthermore influenced the conformation of E71, which led to a distortion of the extracellular entrance of the filter, in resemblance of the inactivated state. ${ }^{36}$ Hence, we hypothesize that in the absence of anionic lipids, the side chains of R64 and R89 relocate and modulate the hydrogen-bonding network behind the selectivity filter, which could be important for KcsA channel activity. This view is further supported by electrophysiological measurements (Figure S6) that suggest a decrease in channel activity with decreasing molar fraction of anionic lipids in KcsA but not in the case KcsA-Kv1.3. Since the transition to the inactivated state of KcsA was proposed to show cooperative behavior, ${ }^{37}$ it could also be imagined that marked cooperative specific lipid binding, which we observe in CGMD simulations, is reflected in channel activity.

Furthermore, our data on KcsA-Kv1.3 suggest that in certain $\mathrm{Kv}$ channels (Figure 1), the formation of an intersubunit saltbridge between S5 and S6 helices diminishes the influence of specific anionic lipid binding to the pore domain on channel activity. Interestingly, an intersubunit salt-bridge in proximity to the selectivity filter reminiscent of D64-R89 in KcsA-Kv1.3 was shown to modulate inactivation in Kir1.1 inward rectifier. ${ }^{38}$ The formation and dissolution of salt-bridge D64-R89 itself may hence be of general functional importance. SsNMR studies of KcsA-Kv1.3 revealed that while residue D64 could be clearly assigned in the conductive state, its resonances could not be assigned in the inactivated state, ${ }^{39}$ presumably due to enhanced mobility or due to conformational rearrangement. We propose that dissolution of salt-bridge D64-R89 in the inactivated state of KcsA-Kv1.3 influences other residues behind the selectivity filter, in analogy to R64 and R89 of KcsA in the absence of negatively charged lipids.

\section{CONCLUSIONS}

We have compared specific lipid binding to potassium channels KcsA and chimeric KcsA-Kv1.3 on the structural and functional level. Taken as a whole, our data demonstrate the functional importance of nonannular anionic lipid binding to KcsA, with the channel's activity correlating markedly with strong, highly specific, and cooperative binding of anionic lipids to the channel's extracellular nonannular site (see also Figure S6). In addition, specific annular lipid binding may be a further origin of the influence of anionic lipids on channel activity. Together, our data provide a framework to derive a molecular mechanism 
of the functional influence of specific lipid binding to KcsA. Moreover, our study demonstrates a reduced functional influence of nonannular lipid binding to the pore domain of KcsA-Kv1.3 and points to a functional role of a salt-bridge between adjacent channel subunits, which may occur in many mammalian $\mathrm{Kv}$ channels.

In our contribution, we exclusively discuss specific lipid binding to the extracellular side of KcsA. Remarkably, in a recent contribution, ${ }^{40}$ using the noninactivating KcsA mutant E71A, a functional role of the cytoplasmic N-terminal helix as a kind of lipid sensor was described, which points to an even more important impact of specific lipid binding on KcsA. We like to stress that an effect of nonannular lipid binding on the inactivation gate, which we infer from our data, is unlikely to be observable with KcsA mutant E71A, the inactivation gate of which is always open. ${ }^{30}$ Note that besides the here discussed influence of direct specific lipid binding to already membraneinserted proteins, specific lipid-protein interactions, and thus the membrane composition, can critically affect membrane protein topogenesis and initial assembly. ${ }^{41,42}$

CGMD simulations overcome the typical length and time scale problem of atomistic simulations, which are usually limited to system sizes and simulation times $<10 \mathrm{~nm}$ and a few hundreds of ns. Notably, the increase in system size and sampling time greatly enhances the potential to compare computational observations to powerful experimental techniques like confocal microscopy or ssNMR. ${ }^{2,43}$ This has been exploited to shed light on intricate phenomena like lipid domain formation or membrane protein lateral distribution. ${ }^{2,44}$ Such processes are notoriously difficult to study experimentally and are likely critically involved in forming membrane protein supramolecular structures and in membrane organization in general. Combining CGMD simulations and ssNMR experiments as shown here allows for the characterization of structural and dynamical features of nonannular lipid binding and of specific lipid-protein interactions in general in a membrane setting and at atomic scale.

\section{EXPERIMENTAL SECTION}

Molecular Dynamics Simulations. Coarse-grained (CG) simulations were carried out using the GROMACS simulations package version $4.5 .3^{45}$ and the MARTINI ${ }^{18,19}$ force field and its extension to proteins, together with an integration step of $25 \mathrm{fs}$ and the standard settings for nonbonded interactions in a NPT ensemble with period boundary conditions. Simulation times were multiplied by a factor 4 to account for the smoothness of the CG potentials. The system was semi-isotropically coupled to a pressure bath at $1 \mathrm{bar}\left(\tau_{\mathrm{p}}=3 \mathrm{ps}\right)$ and coupled $\left(\tau_{\mathrm{T}}=0.3 \mathrm{ps}\right)$ to a heat bath. A solvated mixed DPPC:DPPG (3:1 molar ratio) bilayer, consisting of 1536 DPPC lipids, 512 DPPG lipids, 25088 water, and 512 sodium GC-beads, was self-assembled and equilibrated over $4.4 \mu \mathrm{s}$ at $323 \mathrm{~K}$, which resulted in a large membrane patch with dimensions of $25.2 \times 25.2 \mathrm{~nm}^{2}$. A frame of this system was extracted after $4.4 \mu \mathrm{s}$, and the closed-conductive KcsAKv1.3 channel inserted while keeping the PC:PG 3:1 ratio. This system was equilibrated over $20 \mu$ s while keeping the channel core comprising the filter (backbone and side chain beads) and the intracellular side (backbone beads only), corresponding to residues $22-49,67-82$, and $91-115$, rigid throughout all simulations. Position restraints on the backbone beads of the residues at the membrane/ water interface (residues 50-66 and 83-90) of the extracellular side were gradually reduced during equilibration and very light position restraints of $10 \mathrm{kj} / \mathrm{mol} \cdot \mathrm{nm}^{2}$ were applied during the final runs. KcsA was inserted in two different mixed bilayers extracted after 4.0 and 4.4 $\mu$ s to minimize the influence of the initial lipid distribution. The KcsAKv1.3 CGMD model was converted from an atomic-resolution
ssNMR structure, which will be discussed elsewhere and which was derived from extensive ssNMR measurements in lipid bilayers. ${ }^{17,29}$ All common resolved ssNMR chemical shifts showed very little deviation, which confirmed the conservation of KcsA's fold in KcsA-Kv1.3, so that we could derive KcsA's starting structure by back-mutating KcsAKv1.3 to enhance the comparability of the simulations. Since we noted that a lipid shell of $\sim 3.5 \mathrm{~nm}$ around the channel remained in the liquid phase during all simulation, ${ }^{46}$ even below the phase-transition temperatures of DPPC/PG, we carried out the final runs at $300 \mathrm{~K}$ to be closer to the temperatures used for ssNMR and electrophysiological experiments.

Atomistic simulations were performed with the GROMOS53a6 force field ${ }^{47}$ and the Berger lipid parameters. ${ }^{48}$ The initial coordinates for a hydrated 128 DPPC bilayer were taken from Dr. Tieleman's Web site (moose.bio.ucalgary.ca). This patch was expanded to 512 lipids, minimized, equilibrated over $10 \mathrm{~ns}$ and the closed-conductive KcsA channel (PDB code 3EFF), ${ }^{49}$ truncated to residues 22-115, inserted. ${ }^{50}$ The temperature was kept constant at $320 \mathrm{~K}$. Ions (80 $\mathrm{mM} \mathrm{KCl}$ ) and water molecules as well as lipids and the protein were jointly coupled to the thermostat. The system was first equilibrated in a NVT ensemble, then for $10 \mathrm{~ns}$ in a NPT ensemble using semiisotropic pressure coupling with gradually reduced force constants and finally freely evolved over 35 ns. See Supporting Information for further details.

Sample Preparation. Expression and purification of LVWY reverse-labeled KcsA-Kv1.3 and uniformly labeled KcsA have been described in ref 16. For the ssNMR experiments, reconstitution was performed at a 100:1 lipid/protein molar ratio. For the single channel recordings, unlabeled KcsA and KcsA-Kv1.3 were reconstituted at 1000:1 lipid/protein mass ratio. Both KcsA and KcsA-Kv1.3 were in the closed-conductive state.

SsNMR Spectroscopy. The ssNMR experiments were measured in magnetic fields of 11.7 T. 2D PARIS ${ }^{13} \mathrm{C}-{ }^{13} \mathrm{C}$ spin diffusion experiments $^{24,25}$ (pulse length $=0.5 \tau_{\text {rot }}, 13 \mathrm{kHz}$ recoupling amplitude) were carried out at $20 \mathrm{kHz}$ MAS and $265 \mathrm{~K}$ with mixing times of 30 ms using PISSARRO decoupling. ${ }^{51}$ The rotational resonance ${ }^{23}\left(R^{2}\right)$ experiment was performed at $269 \mathrm{~K}$ and $15 \mathrm{kHz}$ MAS, close to the chemical shift difference of $14.9 \mathrm{kHz}$ between $\mathrm{D} 64 \mathrm{C} \beta$ and R89C $\zeta$. No decoupling was applied during $150 \mathrm{~ms}$ mixing time, to broaden the $R^{2}$ condition, ${ }^{52}$ and SPINAL64 decoupling ${ }^{33}$ was applied during acquisition periods.

Single Channel Measurements. Single channel recordings of KcsA were performed on planar lipid bilayer setup (Compact, Ionovation $\mathrm{GmbH}$ ). Lipid bilayers were formed by painting the lipids dissolved in $\mathrm{n}$-decane over a $200 \mu \mathrm{m}$ hole in a Teflon-septum that separated two chambers (cis and trans). Initially, the trans chamber contained $20 \mathrm{mM} \mathrm{KCl}$, the cis chamber $250 \mathrm{mM} \mathrm{KCl}$, both of which were buffered to $\mathrm{pH} 4.0$ with $10 \mathrm{mM}$ succinic acid. KcsA proteoliposomes $(1-5 \mu \mathrm{L})$ were added to the cis chamber of the planar bilayer system with a micropipet. After channel insertion, recording conditions were established by perfusion to symmetrical conditions. The cis side contained $150 \mathrm{mM} \mathrm{KCl}$ and $10 \mathrm{mM} \mathrm{HEPES}$ at $\mathrm{pH} 7.0$, and the trans side contained $150 \mathrm{mM} \mathrm{KCl}$ and $10 \mathrm{mM}$ succinic acid at $\mathrm{pH}$ 4.0. All measurements were performed at room temperature. Data are sampled at $10 \mathrm{kHz}$ and filtered at $1 \mathrm{kHz}$. Reported data are result of statistical analysis of 3-5 independent experiments.

\section{ASSOCIATED CONTENT}

\section{Supporting Information}

Further details of the simulations. This material is available free of charge via the Internet at http://pubs.acs.org.

\section{AUTHOR INFORMATION}

\section{Corresponding Author}

m.baldus@uu.nl

\section{Notes}

The authors declare no competing financial interest. 


\section{ACKNOWLEDGMENTS}

M.W. acknowledges financial support via a FEBS Long-Term Fellowship. This work was supported by The Netherlands Organization for Scientific Research (NWO) through grants 700.26 .121 and 700.10 .443 (M.B.) as well as 700.56 .442 (A.M.J.J.B.) and 722.012.002 (M.W.). A.P. was supported by the DFG grant (Po137/40-1).

\section{REFERENCES}

(1) Lee, A. G. Biochim. Biophys. Acta 2004, 1666, 62-87.

(2) van den Bogaart, G.; Meyenberg, K.; Risselada, H. J.; Amin, H.; Willig, K. I.; Hubrich, B. E.; Dier, M.; Hell, S. W.; Grubmuller, H.; Diederichsen, U.; Jahn, R. Nature 2011, 479, 552-5.

(3) Zheng, H.; Liu, W.; Anderson, L. Y.; Jiang, Q. X. Nat. Commun. 2011, 2, 250.

(4) Milescu, M.; Bosmans, F.; Lee, S.; Alabi, A. A.; Kim, J. I.; Swartz, K. J. Nat. Struct. Mol. Biol. 2009, 16, 1080-5.

(5) Phillips, R.; Ursell, T.; Wiggins, P.; Sens, P. Nature 2009, 459, 379-85.

(6) Eddy, M. T.; Ong, T. C.; Clark, L.; Teijido, O.; van der Wel, P. C.; Garces, R.; Wagner, G.; Rostovtseva, T. K.; Griffin, R. G. J. Am. Chem. Soc. 2012, 134, 6375-87.

(7) Niemela, P. S.; Miettinen, M. S.; Monticelli, L.; Hammaren, H.; Bjelkmar, P.; Murtola, T.; Lindahl, E.; Vattulainen, I. J. Am. Chem. Soc. 2010, 132, 7574-5.

(8) Domene, C.; Bond, P. J.; Deol, S. S.; Sansom, M. S. J. Am. Chem. Soc. 2003, 125, 14966-7.

(9) Lee, A. G. Biochem. Soc. Trans. 2011, 39, 761-6.

(10) Valiyaveetil, F. I.; Zhou, Y.; MacKinnon, R. Biochemistry 2002, 41, 10771-7.

(11) Marius, P.; Alvis, S. J.; East, J. M.; Lee, A. G. Biophys. J. 2005, 89, 4081-9.

(12) Marius, P.; Zagnoni, M.; Sandison, M. E.; East, J. M.; Morgan, H.; Lee, A. G. Biophys. J. 2008, 94, 1689-98.

(13) Marius, P.; de Planque, M. R.; Williamson, P. T. Biochim. Biophys. Acta 2012, 1818, 90-6.

(14) Miller, C. Genome Biol 2000, 1, reviews0004.

(15) Ramu, Y.; Xu, Y.; Lu, Z. Nature 2006, 442, 696-9.

(16) Lange, A.; Giller, K.; Hornig, S.; Martin-Eauclaire, M. F.; Pongs, O.; Becker, S.; Baldus, M. Nature 2006, 440, 959-62.

(17) Schneider, R.; Ader, C.; Lange, A.; Giller, K.; Hornig, S.; Pongs, O.; Becker, S.; Baldus, M. J. Am. Chem. Soc. 2008, 130, 7427-35.

(18) Marrink, S. J.; Risselada, H. J.; Yefimov, S.; Tieleman, D. P.; de Vries, A. H. J. Phys. Chem. B 2007, 111, 7812-24.

(19) Monticelli, L.; Kandasamy, S. K.; Periole, X.; Larson, R. G.; Tieleman, D. P.; Marrink, S. J. J. Chem. Theory Comput. 2008, 4, 819834.

(20) Deol, S. S.; Domene, C.; Bond, P. J.; Sansom, M. S. Biophys. J. 2006, 90, 822-30.

(21) Huijbregts, R. P.; de Kroon, A. I.; de Kruijff, B. Biochim. Biophys. Acta 2000, 1469, 43-61.

(22) Alvis, S. J.; Williamson, I. M.; East, J. M.; Lee, A. G. Biophys. J. 2003, 85, 3828-38

(23) Raleigh, D. P.; Levitt, M. H.; Griffin, R. G. Chem. Phys. Lett. 1988, 146, 71-76.

(24) Weingarth, M.; Bodenhausen, G.; Tekely, P. J. Am. Chem. Soc. 2009, 131, 13937-9.

(25) Weingarth, M.; Tekely, P.; Bruschweiler, R.; Bodenhausen, G. Chem. Commun. (Cambridge, U. K.) 2010, 46, 952-4.

(26) Jiang, Y.; Lee, A.; Chen, J.; Ruta, V.; Cadene, M.; Chait, B. T.; MacKinnon, R. Nature 2003, 423, 33-41.

(27) Weingarth, M.; Ader, C.; Melquiond, A. J.; Nand, D.; Pongs, O.; Becker, S.; Bonvin, A. M.; Baldus, M. Biophys. J. 2012, 103, 29-37.

(28) Lee, C. W.; Griffin, R. G. Biophys. J. 1989, 55, 355-8.

(29) Nand, D.; Cukkemane, A.; Becker, S.; Baldus, M. J. Biomol. NMR 2012, 52, 91-101.
(30) Cordero-Morales, J. F.; Cuello, L. G.; Zhao, Y.; Jogini, V.; Cortes, D. M.; Roux, B.; Perozo, E. Nat. Struct. Mol. Biol. 2006, 13, 311-8.

(31) Cordero-Morales, J. F.; Jogini, V.; Chakrapani, S.; Perozo, E. Biophys. J. 2011, 100, 2387-93.

(32) Cuello, L. G.; Jogini, V.; Cortes, D. M.; Perozo, E. Nature 2010, 466, 203-8.

(33) Ader, C.; Schneider, R.; Hornig, S.; Velisetty, P.; Wilson, E. M.; Lange, A.; Giller, K.; Ohmert, I.; Martin-Eauclaire, M. F.; Trauner, D.; Becker, S.; Pongs, O.; Baldus, M. Nat. Struct. Mol. Biol. 2008, 15, 60512.

(34) Cordero-Morales, J. F.; Cuello, L. G.; Perozo, E. Nat. Struct. Mol. Biol. 2006, 13, 319-22.

(35) Cordero-Morales, J. F.; Jogini, V.; Lewis, A.; Vasquez, V.; Cortes, D. M.; Roux, B.; Perozo, E. Nat. Struct. Mol. Biol. 2007, 14, 1062-9.

(36) Berneche, S.; Roux, B. Structure 2005, 13, 591-600.

(37) Rotem, D.; Mason, A.; Bayley, H. J. Gen. Physiol. 2009, 135, 2942.

(38) Sackin, H.; Nanazashvili, M.; Li, H.; Palmer, L. G.; Walters, D. E. Biophys. J. 2009, 97, 1058-66.

(39) Ader, C.; Schneider, R.; Hornig, S.; Velisetty, P.; Vardanyan, V.; Giller, K.; Ohmert, I.; Becker, S.; Pongs, O.; Baldus, M. EMBO J. 2009, $28,2825-34$

(40) Iwamoto, M.; Oiki, S. Proc. Natl. Acad. Sci. U.S.A. 2013, 110, 749-54.

(41) Bogdanov, M.; Heacock, P.; Guan, Z.; Dowhan, W. Proc. Natl. Acad. Sci. U.S.A. 2010, 107, 15057-62.

(42) Bogdanov, M.; Xie, J.; Dowhan, W. J. Biol. Chem. 2009, 284, 9637-41.

(43) Vostrikov, V. V.; Hall, B. A.; Greathouse, D. V.; Koeppe, R. E., 2nd; Sansom, M. S. J. Am. Chem. Soc. 2010, 132, 5803-11.

(44) Schafer, L. V.; de Jong, D. H.; Holt, A.; Rzepiela, A. J.; de Vries, A. H.; Poolman, B.; Killian, J. A.; Marrink, S. J. Proc. Natl. Acad. Sci. U.S.A. 2011, 108, 1343-8.

(45) Hess, B.; Kutzner, C.; van der Spoel, D.; Lindahl, E. J. Chem. Theory Comput. 2008, 4, 435-447.

(46) Periole, X.; Knepp, A. M.; Sakmar, T. P.; Marrink, S. J.; Huber, T. J. Am. Chem. Soc. 2007, 134, 10959-65.

(47) Soares, T. A.; Hunenberger, P. H.; Kastenholz, M. A.; Krautler, V.; Lenz, T.; Lins, R. D.; Oostenbrink, C.; van Gunsteren, W. F. J. Comput. Chem. 2005, 26, 725-37.

(48) Berger, O.; Edholm, O.; Jahnig, F. Biophys. J. 1997, 72, 200213.

(49) Uysal, S.; Vasquez, V.; Tereshko, V.; Esaki, K.; Fellouse, F. A.; Sidhu, S. S.; Koide, S.; Perozo, E.; Kossiakoff, A. Proc. Natl. Acad. Sci. U.S.A. 2009, 106, 6644-9.

(50) Kandt, C.; Ash, W. L.; Tieleman, D. P. Methods 2007, 41, 47588.

(51) Weingarth, M.; Tekely, P.; Bodenhausen, G. Chem. Phys. Lett. 2008, 466, 247-251.

(52) Peng, X. H.; Libich, D.; Janik, R.; Harauz, G.; Ladizhansky, V. J. Am. Chem. Soc. 2008, 130, 359-369.

(53) Fung, B. M.; Khitrin, A. K.; Ermolaev, K. J. Magn. Reson. 2000, $142,97-101$. 